\title{
Artificial Intelligence and Acute Stroke Imaging
}

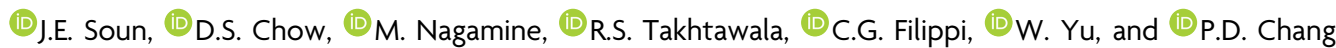

\begin{abstract}
SUMMARY: Artificial intelligence technology is a rapidly expanding field with many applications in acute stroke imaging, including ischemic and hemorrhage subtypes. Early identification of acute stroke is critical for initiating prompt intervention to reduce morbidity and mortality. Artificial intelligence can help with various aspects of the stroke treatment paradigm, including infarct or hemorrhage detection, segmentation, classification, large vessel occlusion detection, Alberta Stroke Program Early CT Score grading, and prognostication. In particular, emerging artificial intelligence techniques such as convolutional neural networks show promise in performing these imaging-based tasks efficiently and accurately. The purpose of this review is twofold: first, to describe Al methods and available public and commercial platforms in stroke imaging, and second, to summarize the literature of current artificial intelligence-driven applications for acute stroke triage, surveillance, and prediction.
\end{abstract}

ABBREVIATIONS: $\mathrm{Al}=$ artificial intelligence; $\mathrm{ANN}=$ artificial neural network; $\mathrm{AUC}=$ area under the curve; $\mathrm{CNN}=$ convolutional neural network; $\mathrm{DL}=$ deep learning; ICC = intraclass correlation coefficient; ICH = intracranial hemorrhage; $L V O=$ large vessel occlusion; $M L=$ machine learning; MRP = MR perfusion; $\mathrm{RF}=$ random forest; $\mathrm{SVM}=$ support vector machine

S

troke is the second leading cause of death worldwide with an annual mortality of about 5.5 million. ${ }^{1,2}$ In the United States, nearly 800,000 people have a stroke annually, and the economic burden of stroke is estimated at $\$ 34$ billion per year. ${ }^{3}$ Morbidity is high, with more than half of patients with stroke left chronically disabled. ${ }^{2}$ Neuroimaging is an important tool for the detection, characterization, and prognostication of acute strokes, including ischemic and hemorrhagic subtypes. Artificial intelligence (AI) technology is a rapidly burgeoning field, providing a promising avenue for fast and efficient imaging analysis. ${ }^{4}$ AI applications for imaging of acute cerebrovascular disease have been implemented, including tools for triage, quantification, surveillance, and prediction. This review aims to summarize the current landscape of AIdriven applications for acute cerebrovascular disease assessment focusing primarily on deep learning (DL) methods.

Received April 30, 2020; accepted after revision July 22.

From the Departments of Radiological Sciences (J.E.S., D.S.C., P.D.C.) and Neurology (M.N., W.Y.), Center for Artificial Intelligence in Diagnostic Medicine (D.S.C., R.S.T., P.D.C.), University of California, Irvine, Orange, California, and the Department of Radiology (C.G.F.), Northwell Health, Lenox Hill Hospital, New York, New York.

Please address correspondence to Daniel S. Chow, MD, Department of

Radiological Sciences, UC Irvine Health, Douglas Hospital, Route 140, Rm 0115, Orange, CA 92868; e-mail: chowd3@hs.uci.edu; @TheCAIDM

- Indicates open access to non-subscribers at www.ajnr.org

Indicates article with supplemental on-line tables.

http://dx.doi.org/10.3174/ajnr.A6883

\section{OVERVIEW OF AI}

Although AI, machine learning (ML), and DL are used interchangeably, these in fact represent subdisciplines. Specifically, DL is a subset of ML, and ML is a subset of AI (Fig 1). Broadly, AI uses computers to perform tasks that typically require human knowledge. ML, a subset of AI, uses statistical approaches to enable machines to optimize outcome prediction as they are exposed to data and train computers for pattern recognition, a task generally requiring human intelligence. ${ }^{5} \mathrm{ML}$ offers several potential advantages over visual inspection by human experts, including objective and quantitative evaluation, the ability to detect subtle voxel-level patterns, speed, and large-scale implementation. Feature selection, classifier type, and DL are key considerations for the application of ML techniques to imaging.

\section{Feature Selection}

Just as a radiologist summarizes an image with a few key descriptors (eg, hemorrhage volume), ML algorithms attempt to do the same with a matrix of voxels. Different feature selection methods can identify a subset of variables to develop a predictive model. Selecting relevant features is important for the explainability, speed, and cost efficiency of a model and to avoid overfitting. ${ }^{6}$

\section{Classifier Type}

After each image is converted into numeric descriptors, a method is chosen to leverage this information to predict 1 of multiple potential classes. For certain cases, even very simple models such 
as basic logistic or linear regression could be effective. ${ }^{7}$ However, if nonindependent, nonlinear relationships are expected between the various chosen features, a more complex model is required. Many such ML classifiers exist, and the most popular include random forest (RF), support vector machine (SVM), k-nearest neighbor clustering, and neural networks. ${ }^{8}$ In general, these techniques are modeled by an underlying finite number of adjustable parameters. As a given set of features is passed through the model, these adjustable parameters act to convert the input descriptors into a predicted output class. Starting with randomly initialized parameters, a series of iterative updates is performed until an accurate mapping between numeric features and correct class is achieved, thus "training" the ML model. ${ }^{9}$

\section{Deep Learning}

DL through neural networks is distinguished by the ability to independently learn abstract, high-order features from data without requiring feature selection. Artificial neural networks (ANNs) are a subtype of DL that mimic biologic neurons and are composed of an input, 1 or more hidden layers, and an output. Generally, in computer vision, convolutional neural networks (CNNs) are most successful and popular for image classification in medical imaging. $\mathrm{CNNs}$ represent all recent winning entries within the annual ImageNet Classification challenge, consisting of more than 1 million photographs in 1000 object categories, with a 3.6\% classification error rate. ${ }^{10,11} \mathrm{CNNs}$ are distinguished from traditional ML approaches by automatically identifying patterns in complex imaging datasets, thus combining both feature selection and classification into 1 algorithm and removing the need for direct human interaction during the training process. Recent advances in CNNs have achieved human accuracy in identification of everyday objects such as cats and dogs, which had previously been impossible to model using rigid mathematical formulas. ${ }^{12} \mathrm{CNNs}$ have already shown promise in the detection of pulmonary nodules, ${ }^{13}$ colon cancer, ${ }^{14}$ and cerebral microbleeds. ${ }^{15}$

\section{EVALUATION OF AI PERFORMANCE}

Table 1 details performance metrics and limitations of AI methods.

\section{Accuracy}

It is imperative that evaluation of ML models assess the accuracy of algorithms. Often, when testing large numbers of potential features, a few numeric descriptors meet the threshold for statistical significance between 2 target classes. However, $P$ values are more often a reflection of the underlying power (sample size) of an experiment and may or may not relate to the clinical significance of the identified difference in features. As a result, it is critical not only to prove that a difference in features exists but also to assess the sensitivity, specificity, and accuracy of the feature(s) to predict a given end point. For classification, receiver operating characteristic curves can evaluate a model's performance, with the area under the curve (AUC) representing an aggregate measure for performance across all possible classification thresholds of a receiver operating characteristic curve. For segmentation analysis, Dice similarity coefficients and Pearson correlation coefficients are typically used. The Dice score measures the spatial overlap between the manually segmented and neural networkderived segmentations. Dice scores range from 0 (no overlap) to 1 (perfect overlap) and are commonly used to evaluate segmentation performance. ${ }^{16}$

\section{Limitations}

ML and DL approaches have limitations that should be considered. First, the development of algorithms requires data sets that are large, organized, well-classified, and accurate. Interpretability is challenging, especially for DL algorithms. To mitigate this "black box"

FIG 1. Al uses computers to mimic human intelligence. $M L$ is a subset of $A l$, and $D L$ is a subset of ML.

(Examples: Artificial Neural Network, Convolutional Neural Network, etc.)

\section{Table 1: Machine learning performance metrics and limitations}

\begin{tabular}{|c|c|c|}
\hline Performance metrics & Segmentation & $\begin{array}{l}\text { Sensitivity (recall): TP/(TP }+ \text { FN) } \\
\text { Specificity (true-negative rate): TN/(TN }+ \text { FP) } \\
\text { Accuracy: number of correct predictions/total predictions } \\
\text { AUC: plot of true positive rate (sensitivity) against false positive rate }(1-\text { specificity) } \\
\text { Dice similarity coefficient: overlap of } 2 \text { samples } \\
\text { Pearson correlation coefficient: strength of linear relationship between } 2 \text { variables }\end{array}$ \\
\hline $\begin{array}{l}\text { Limitations and ways to } \\
\text { address them }\end{array}$ & $\begin{array}{l}\text { Requires large } \\
\text { Interpretability } \\
\text { Overfitting: mc }\end{array}$ & $\begin{array}{l}\text { ts: multisite collaboration, open-source datasets } \\
\text { cy maps } \\
\text { ining data, regularization, and batch normalization }\end{array}$ \\
\hline
\end{tabular}

Note:-FP indicates false positive; FN, false-negative; ROC, receiver operating characteristic; TN, true-negative; TP, true positive. 
Table 2: Open-source datasets for stroke and hemorrhage

\begin{tabular}{|c|c|c|c|c|}
\hline Dataset & $\begin{array}{c}\text { Cerebrovascular } \\
\text { Disease }\end{array}$ & Annotated Data & Number of Scans & $\begin{array}{l}\text { Imaging } \\
\text { Technique }\end{array}$ \\
\hline $\begin{array}{l}\text { Anatomical Tracings of } \\
\text { Lesions After Stroke } \\
\text { (ATLAS) }^{19}\end{array}$ & $\begin{array}{l}\text { Subacute or chronic } \\
\text { ischemic strokes }\end{array}$ & Manually segmented stroke lesions & 304 & $\begin{array}{l}\text { T1-weighted MR } \\
\text { imaging }\end{array}$ \\
\hline $\mathrm{CQ} 500^{20^{\circ}}$ & Hemorrhage & $\begin{array}{l}\text { Hemorrhage, subtype, location, and } \\
\text { associated hemorrhage findings }\end{array}$ & 491 & $\mathrm{CT}$ \\
\hline $\begin{array}{l}\text { RSNA Brain Hemorrhage } \\
\text { CT Dataset }{ }^{21}\end{array}$ & Hemorrhage & Hemorrhage, subtypes & 874,035 & $\mathrm{CT}$ \\
\hline $\begin{array}{l}\text { Ischemic Stroke Lesion } \\
\text { Segmentation (ISLES) } \\
\text { 2016-2017 }\end{array}$ & Ischemic stroke & $\begin{array}{l}\text { Perfusion and diffusion MR imaging } \\
\text { of patients with stroke and clinical } \\
\text { outcomes }\end{array}$ & $\begin{array}{l}35 \text { training and } 19 \text { testing } \\
\text { (2016); } 43 \text { training and } 32 \\
\text { testing (2017) }\end{array}$ & $\begin{array}{l}\text { MR imaging, } \\
\text { MRP }\end{array}$ \\
\hline ISLES $2018^{23}$ & Ischemic stroke & $\begin{array}{l}\text { CT and perfusion of patients with } \\
\text { stroke }\end{array}$ & $\begin{array}{l}94 \text { labeled training images } \\
\text { and } 62 \text { unlabeled testing } \\
\text { images }\end{array}$ & CT, CTP \\
\hline
\end{tabular}

Note:-MRP indicates MR perfusion.

Table 3: Commercially available software platforms and their applications ${ }^{\mathrm{a}}$

\begin{tabular}{|c|c|c|c|}
\hline Software & Applications & Machine Learning Algorithm & Imaging Technique \\
\hline \multirow[t]{3}{*}{ Aidoc } & $\mathrm{ICH}$ : identifies $\mathrm{ICH}$, triage, and notification & DL & $\mathrm{CT}$ \\
\hline & LVO: identifies LVO, triage, and notification & DL & CTA \\
\hline & CTP: orchestration of third-party perfusion results & Other & CTP \\
\hline \multirow[t]{3}{*}{ Avicenna.Al } & CINA ICH: identifies ICH, triage, and notification & DL & $\mathrm{CT}$ \\
\hline & CINA LVO: identifies LVO, triage, and notification & DL & CTA \\
\hline & CINA ASPECTS: ASPECTS scoring; provides heat map & DL & $\mathrm{CT}$ \\
\hline \multirow[t]{5}{*}{ Brainomix } & e-Blood: identifies and quantifies ICH volume with mask overlay & $\mathrm{DL}$ & $\mathrm{CT}$ \\
\hline & $\begin{array}{l}\text { e-ASPECTS: identifies ASPECTS, voxelwise map of early ischemic } \\
\text { change, and core infarct volume }\end{array}$ & Predominantly ML & CT \\
\hline & $\begin{array}{l}\text { e-CTA: identifies and notifies LVO, collateral score, and collateral } \\
\text { vessel attenuation; voxelwise map of collateral deficit }\end{array}$ & $\begin{array}{l}\text { Combination of } \mathrm{DL} \text { and } \\
\text { traditional } \mathrm{ML}\end{array}$ & CTA \\
\hline & e-ASPECTS HDVS: identifies and measures hyperattenuated vessel & $\mathrm{DL}$ & CT \\
\hline & e-Mismatch: identifies mismatch on CTP and MR imaging & Deconvolution & CTP, MR imaging, MRP \\
\hline \multirow[t]{5}{*}{ RapidAl } & Rapid ICH: identifies and classifies ICH & $\mathrm{DL}$ & CT \\
\hline & Rapid ASPECTS: identifies ASPECTS, measurement, and scoring & RF & $\mathrm{CT}$ \\
\hline & $\begin{array}{l}\text { Rapid CTA: identifies and notifies LVO and collateral vessel } \\
\text { attenuation }\end{array}$ & Other & CTA \\
\hline & $\begin{array}{l}\text { Rapid CTP: identifies mismatch on CTP, collateral maps, and } \\
\text { scoring }\end{array}$ & Other & CTP \\
\hline & Rapid MR: identifies mismatch on MR, collateral maps, and scoring & Other & MR imaging, MRP \\
\hline \multirow[t]{3}{*}{ Viz.ai } & Viz ICH: identifies and triages $\mathrm{ICH}$ & DL & $\mathrm{CT}$ \\
\hline & Viz LVO: identifies and triages LVO & $\mathrm{DL}$ & CTA \\
\hline & Viz CTP: automated perfusion color maps and calculations & $\mathrm{DL}$ & CTP \\
\hline
\end{tabular}

Note:-HDVS indicates hyperattenuated vessel sign.

a Some, but not all, of these products have FDA, European, and/or worldwide regulatory clearance at the time of publication.

effect, explainable AI models incorporate tools such as saliency maps. Overfitting is a limitation for ML, when a model mistakenly learns the "noise" instead of the "signal" in a training dataset and thus does poorly with unseen data and is limited in generalizability. ${ }^{17}$ More training data, regularization, and batch normalization are ways to mitigate overfitting. Differences in image acquisition and data storage among institutions and difficulties in sharing data are obstacles to collecting enough data to obtain useful models. Standardization of imaging methods and open-source data collection can address this issue. Additionally, several proprietary ML software platforms have recently been introduced in the market that incorporate various aspects of the stroke pathway into their algorithms; however, comparison and validation of their performance are still necessary to ensure their robustness in routine use. ${ }^{18}$ Despite limitations, ML remains a powerful tool for detection and management of stroke and hemorrhage.

\section{AI PLATFORMS IN STROKE AND HEMORRHAGE Open-Source Datasets}

Large datasets are required for ML algorithms to perform optimally. However, the availability of high-quality large-scale data remains a challenge given barriers in data sharing across institutions, the complexity of building imaging processing pipelines, and the time and cost of data annotation. To address these challenges, many publicly available imaging datasets are now available for ML in stroke (Table 2). ${ }^{19-24}$ These datasets are valuable because they are already anonymized, postprocessed, and annotated, and they can be used for testing and comparing algorithms in diagnosing ischemic stroke and hemorrhage. Many of these datasets are initiated as AI challenges such as the RSNA (Radiology Society of North America) Head CT Challenge for Hemorrhage, ASFNR (American Society of Functional Neuroradiology) Head CT Challenge for Ischemic and Hemorrhagic Stroke, and ISLES 


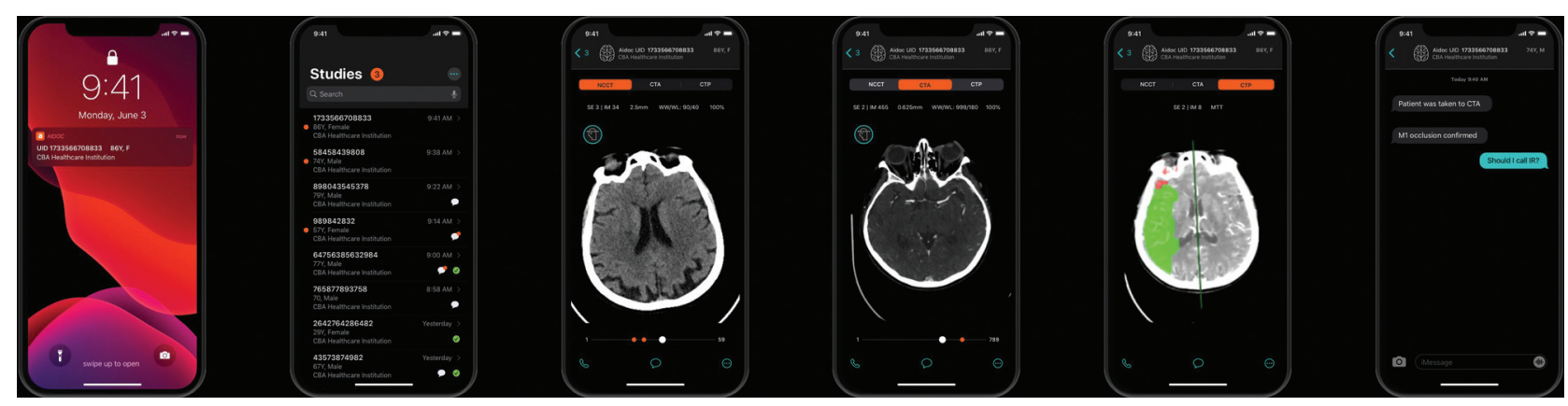

FIG 2. Aidoc stroke triage mobile interface. From left to right, a notification alert, a study list of cases, NCCT of an acute stroke, CTA of an LVO of the right MCA, CTP mean transit time in the right MCA territory, and a text messaging system. Images courtesy of Aidoc.

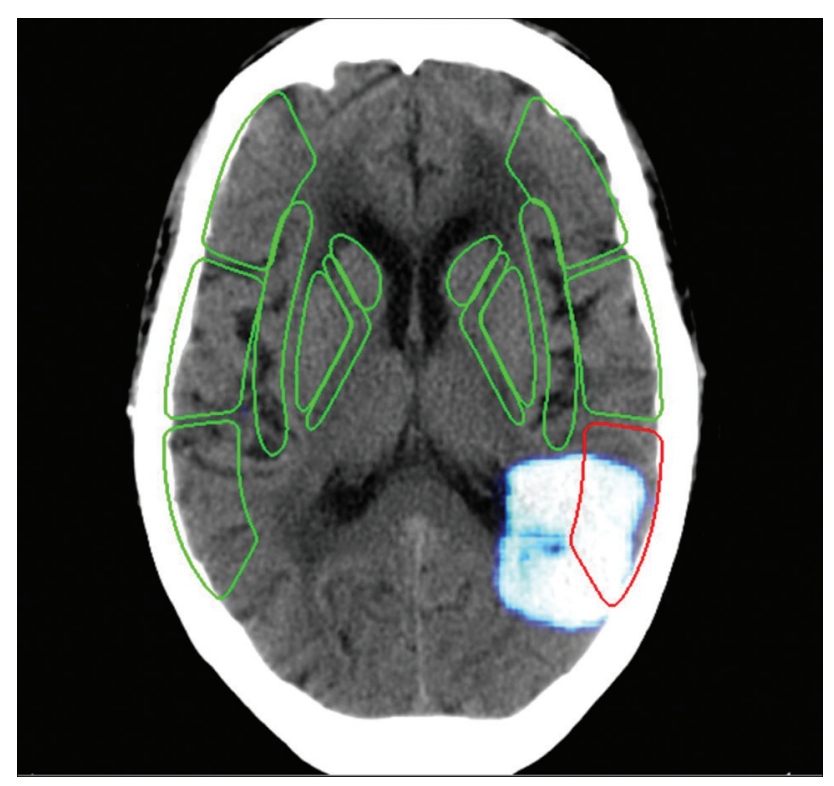

FIG 3. Avicenna.AI DL-based ASPECTS tool demonstrating identification of ASPECTS and a heat map overlay (white). Image courtesy of Avicenna.Al.

(Ischemic Stroke Lesion Segmentation) Challenge for Ischemic Stroke, supporting worldwide collaboration and new algorithm development.

\section{Commercially Available Software Platforms}

Increasingly, commercially available platforms providing automated information about various components of the acute stroke triage pathway are being integrated into routine clinical practice and clinical trials. ${ }^{25-28}$ These tools offer fast and efficient analyses that seek to optimize the delivery of stroke care at spoke and hub hospitals and reduce turnaround times in the clinical workflow. ${ }^{29}$ Table 3 lists some of the most popular commercially available stroke platforms and highlights their capabilities and AI-based algorithms. Figs 2-6 show the various web and mobile interfaces of these software platforms.

\section{AI EVALUATION OF ISCHEMIC STROKE}

Online Tables 1-4 provide an overview of the AI-based models of evaluating ischemic stroke discussed in this section, including detection and core infarct segmentation, identification of large- vessel occlusion (LVO), Alberta Stroke Program Early CT Score (ASPECTS) grading and additional factors in treatment selection, and prognostication.

\section{Detection Methods}

Rapid detection of ischemic infarction is important for triaging patients as potential candidates for thrombolysis because of the narrow window of therapeutic efficacy. Several studies have used ML algorithms for identification of ischemic infarction on CT or MR imaging.

Tang et $\mathrm{al}^{30}$ developed a computer-automated detection (CAD) scheme using a circular adaptive region of interest (CAROI) method on noncontrast head CT to detect subtle changes in attenuation in patients with ischemic stroke. They found that $\mathrm{CAD}$ improved detection of stroke for emergency physicians and radiology residents (AUC of 0.879 improved to 0.942 for emergency physicians and AUC of 0.965 improved to 0.990 for radiology residents) but did not improve significantly detection for experienced radiologists who already had high stroke detection rates. ${ }^{30}$ Another study showed that an ANN was able to distinguish acute stroke from stroke mimics within 4.5 hours of onset (which was verified by clinical and CT and MR imaging data), with a mean sensitivity of $80.0 \%$ and specificity of $86.2 \%{ }^{31}$

\section{Core Infarct Volume Segmentation}

Establishing infarct volumes is important to triage patients for appropriate therapy. AI has been able to establish core infarct volumes on DWI through automatic lesion segmentation. For example, 1 study used an ensemble of 2 CNNs to segment DWI lesions of any size and remove false positives. ${ }^{32}$ This combined CNN approach had a Dice score of 0.61 for small lesions $(<37$ pixel size) and 0.83 for large lesions and outperformed other $\mathrm{CNNs}^{32}$ Guerrero et $\mathrm{al}^{33}$ developed a CNN (uResNet) that segmented and differentiated white matter hyperintensities (WMHs) caused by chronic small-vessel disease from cortically or subcortically based strokes. The uResNet CNN mean Dice scores were 0.7 for white matter hyperintensities and 0.4 for strokes. ${ }^{33}$ The uResNet slightly outperformed the DeepMedic CNN in distinguishing white matter hyperintensities and strokes compared with expert analysis ( $R^{2}$ values 0.951 and 0.791 for white matter hyperintensities and strokes, respectively, using uResNet and 0.942 and 0.688 using DeepMedic). ${ }^{33}$ One limitation of the study was the reliance on FLAIR and T1 images that do not fully account for 


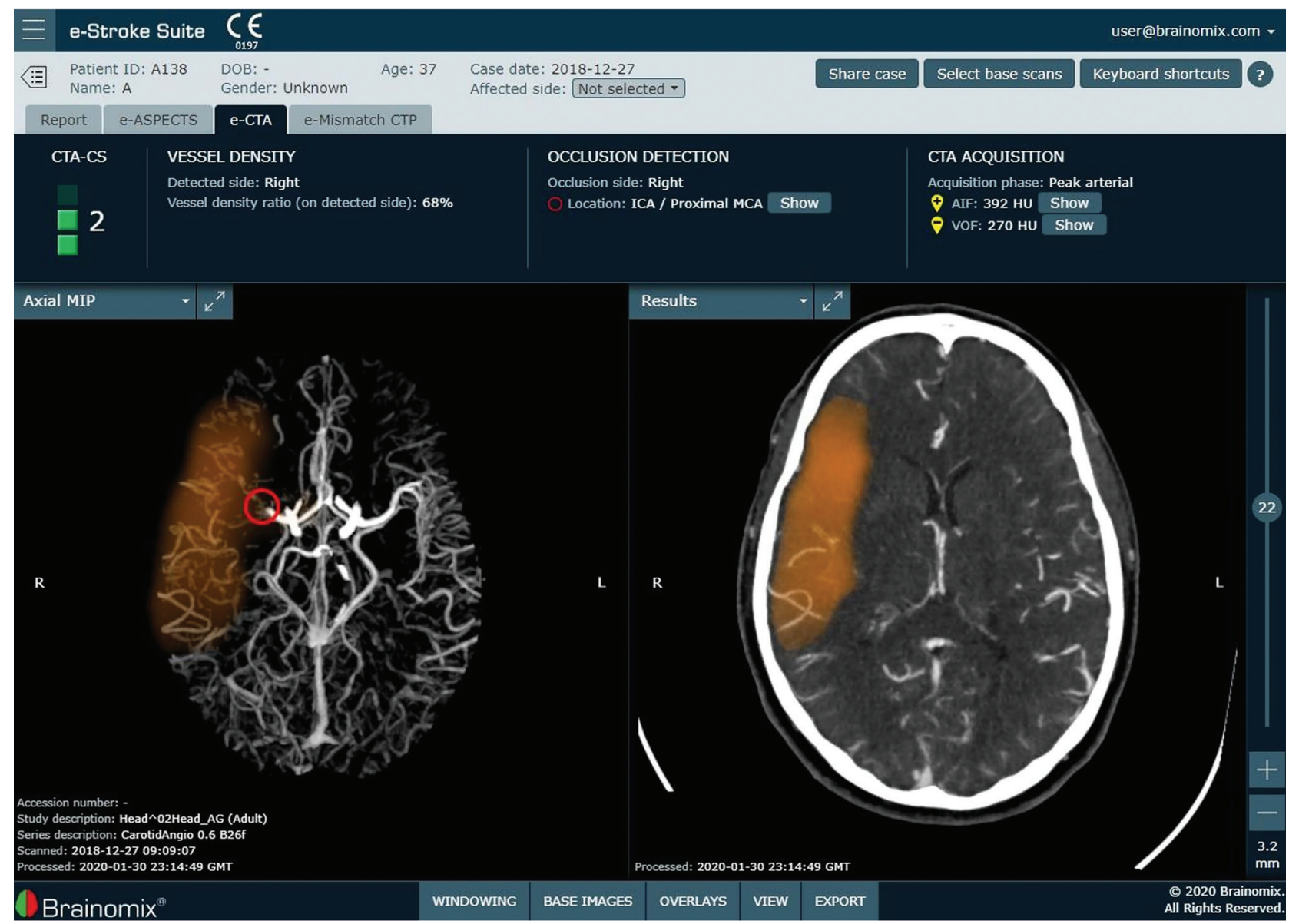

FIG 4. Brainomix e-CTA tool demonstrating identification and localization of an LVO of the right MCA, collateral score and collateral vessel attenuation, and a heat map of the collateral deficit (orange). Images courtesy of Brainomix.

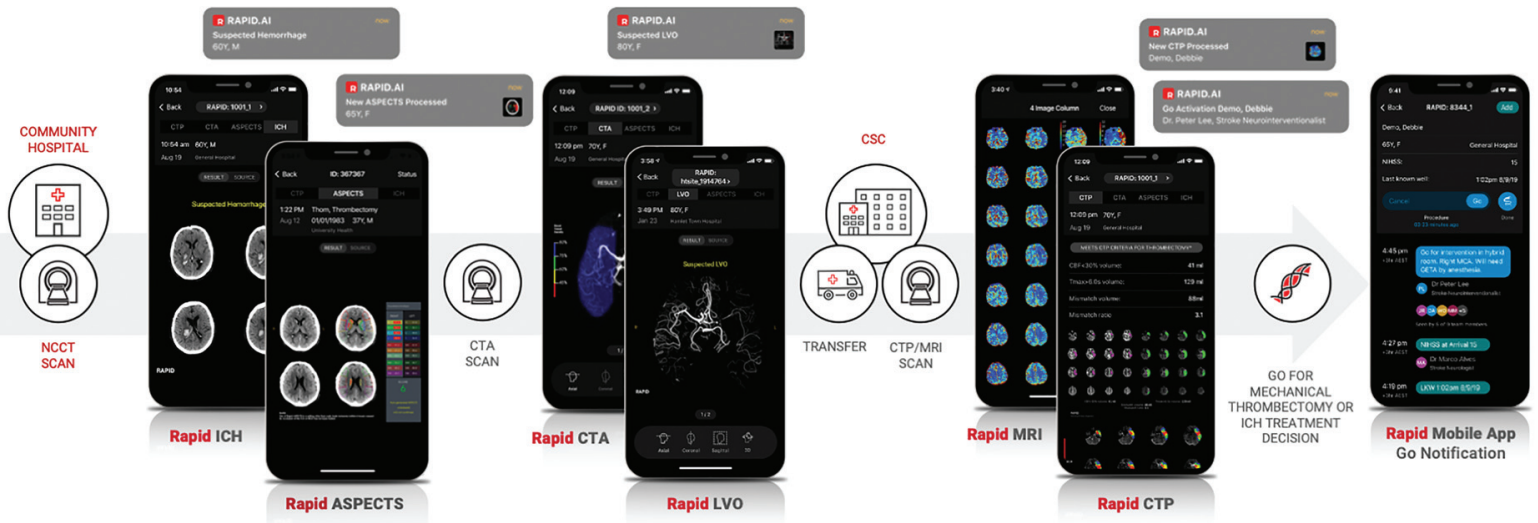

FIG 5. The RapidAI stroke triage or transfer mobile interface, which integrates the hub and spoke model. From left to right, ICH and ASPECTS scoring and alerts on NCCT, LVO detection on CTA, perfusion mismatch on MR imaging or CTP with FDA mechanical thrombectomy indication, and a mobile communication platform with "GO" notification system for rapid treatment decision making. Images courtesy of RapidAl.

timing of stroke occurrence, and the value of uResNet in detection of acute strokes needs evaluation. The first study to use a DL approach on CTA source images to detect acute middle cerebral artery ischemic stroke, a 3D CNN (DeepMedic), performed with a sensitivity of 0.93 , specificity of 0.82 , AUC of 0.93 , and Dice score of $0.61 .^{34}$ Specificity was maximized when the contralateral cerebral hemisphere on CTA was included, and a marginal reduction in false positives was seen when NCCT was included in the algorithm. ${ }^{34}$ Limitations of this $\mathrm{CNN}$ were its tendency to overestimate the volume of small infarcts and underestimate large infarcts compared with manual segmentation by expert radiologists and difficulty in distinguishing old versus new strokes. ${ }^{34}$ 


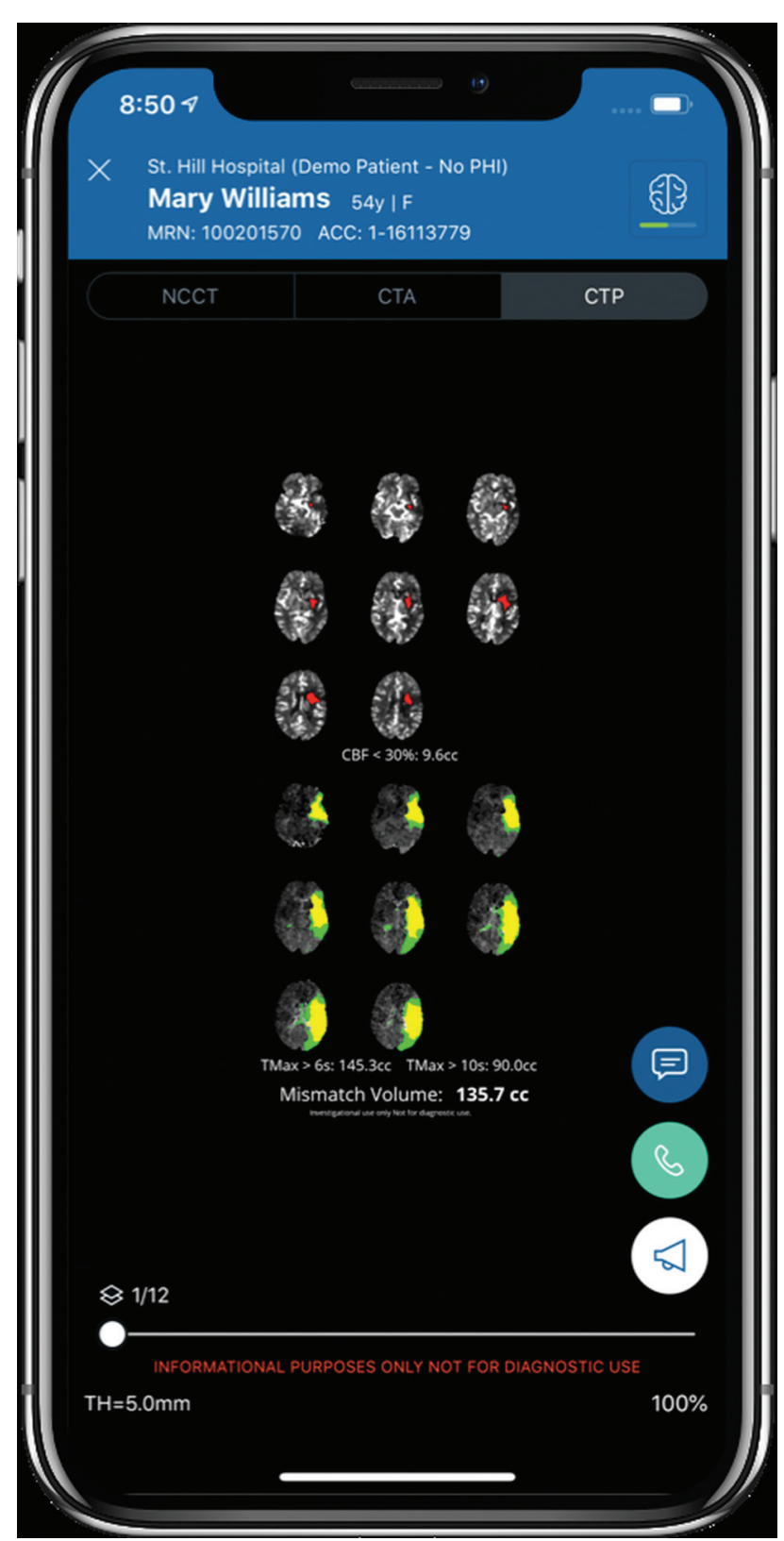

FIG 6. Viz.ai mobile interface showing a left MCA territory infarction with mismatch on CTP. Image courtesy of Viz.ai.

The largest cohort using CTP for core infarct determination based on an ANN was able to accurately identify core infarct volume $(\mathrm{AUC}=0.85$; sensitivity $=0.9$; specificity $=0.62)$ and was not significantly different from a model incorporating clinical data $(\mathrm{AUC}=0.87$; sensitivity $=0.91$; specificity $=0.65) .{ }^{35}$ Although the study minimized the time between CTP and MR imaging DWI reference standard acquisition, any time delay between the CTP and MR imaging may have limited accurate core infarct determination because of core expansion or reversal. A model incorporating a U-net architecture CNN and RF classifier segmented acute ischemic stroke on NCCT with high concordance with manually segmented DWI core volumes $(r=0.76$, $P<.001)$ and manually segmented DWI ASPECTS scores $(r=$ $-0.65, P<.001)$. Furthermore, the agreement approached significance when dichotomizing infarcts using a volume threshold of
$70 \mathrm{~mL}$ (McNemar test, $P=.11$ ). Discrepancies in volumes were attributed to nondetectable early ischemic findings, partial volume averaging, and stroke mimics on CT. ${ }^{36}$

\section{Large Vessel Occlusion}

Diagnosing LVO is essential for identifying candidates who could potentially benefit from mechanical thrombectomy. On NCCT, an SVM algorithm detected the MCA dot sign in patients with acute stroke with high sensitivity (97.5\%). ${ }^{37}$ A neural network that incorporated various demographic, imaging, and clinical variables in predicting LVO outperformed or equaled most other prehospital prediction scales with an accuracy of $0.820 .{ }^{38} \mathrm{~A}$ CNN-based commercial software, Viz-AI-Algorithm v3.04, detected proximal LVO with an accuracy of $86 \%$, sensitivity of $90.1 \%$, specificity of 82.5 , AUC of $86.3 \%$ (95\% CI, $0.83-0.90 ; P \leq$ $.001)$, and intraclass correlation coefficient (ICC) of $84.1 \%$ (95\% CI, $0.81-0.86$; $P \leq .001$ ), and Viz-AI-Algorithm v4.1.2 was able to detect LVO with high sensitivity and specificity ( $82 \%$ and $94 \%$, respectively). ${ }^{39,40}$ No study has yet shown whether AI methods can accurately identify other potentially treatable lesions such as M2, intracranial ICA, and posterior circulation occlusions.

\section{ASPECTS Grading}

ASPECTS is a widely used clinical grading system for assessing extent of early ischemic stroke on NCCT and has been used in randomized clinical trials to select thrombectomy candidates. ${ }^{26,41,42}$ However, grading can be challenging, and interobserver agreement is variable. One commercial software platform with automated ASPECTS scoring (e-ASPECTS, Brainomix) performed as well as neuroradiologists when scoring ASPECTS on NCCT in patients with acute stroke $(P<.003) .{ }^{43}$ However, eASPECTS did not perform as well as neuroradiologists when scoring ASPECTS in patients with acute stroke with baseline non-normal-appearing CT (eg, leukoencephalopathy, old infarcts, or other parenchymal defects), demonstrating a correlation coefficient of 0.59 versus $0.71-0.80$ for experts. ${ }^{44}$ One study found that an automated ASPECTS detection algorithm on NCCT using texture feature extraction to train a RF classifier generated ASPECTS values that had high agreement with expertgenerated DWI ASPECTS scores (ICC $=0.76$ and $\kappa=0.6$ when used for all 10 ASPECTS regions). ${ }^{45}$

Another commercial software platform with automated ASPECTS scoring (Rapid ASPECTS, version 4.9; iSchemaView) showed higher agreement with a consensus ASPECTS grade that takes into account follow-up DWI $(\kappa=0.9)$ compared with neuroradiologists' moderate agreement $(\kappa=0.56-0.57)$, and the software performed well in the immediate time interval 1 hour after stroke onset $(\kappa=0.78)$ and even better 4 hours after stroke onset $(\kappa=0.92){ }^{46}$ This platform had better agreement of ASPECTS grading with DWI infarct volume in patients with large hemispheric infarct compared with experienced readers (median DWI ASPECTS, 3 [IQR, 2-4]; Rapid ASPECTS, 3 [1-6]; and CT ASPECTS for the clinicians, 5 [4-7]. ${ }^{47}$

\section{Additional Factors in Treatment Selection}

Various factors, including collaterals, penumbra, and stroke onset time, are important for evaluating potentially salvageable tissue 
and determining treatment eligibility. An automated commercial software program (e-CTA; Brainomix) combining deep and traditional ML techniques for CTA collateral status determination improved consensus scoring among expert neuroradiologists compared with visual inspection alone, with an ICC of 0.58 (0.46-0.67) improving to $0.77(0.66-0.85 ; P=.003) .{ }^{48}$ Penumbra prediction on a noncontrast $\mathrm{MR}$ imaging pseudocontinuous arterial spin labeling technique using a DL model performed well $($ AUC $=0.958) .{ }^{49}$ This algorithm outperformed traditional ML algorithms and was able to predict endovascular treatment eligibility based on DEFUSE 3 (Endovascular Therapy Following Imaging Evaluation for Ischemic Stroke) trial criteria. Another study evaluating various traditional ML models in predicting stroke onset time demonstrated that incorporation of DL features to the models improved AUC compared with the ground truth (ie, a DWI-FLAIR mismatch), with the optimal AUC of 0.765 incorporating logistic regression and DL features of MR imaging and MR perfusion (MRP) images. ${ }^{50}$ Lee et $\mathrm{al}^{51}$ used DWI-FLAIR mismatch to predict stroke onset time $<4.5$ hours and found that traditional ML models were more sensitive than stroke neurologists (sensitivity $=48.5 \%$ for stroke neurologists vs $75.8 \%$ for logistic regression; $P=.020 ; 72.7 \%$ for SVM, $P=.033 ; 75.8 \%$ for $\mathrm{RF}, P=.013)$.

\section{Prognostication}

Various ML algorithms have been used to predict imaging and clinical outcomes after ischemic stroke. An early classical ML study found that a generalized linear model combining DWI and perfusion-weighted imaging MR images was better than DWI $(P=.02)$ or PWI $(P=.04)$ alone at predicting voxelwise tissue outcomes. ${ }^{52}$ A CNN-based patch sampling of the Tmax feature on MRP outperformed a single voxel-based regression model in predicting final infarct volume, with a mean accuracy of $85.3 \pm$ 9.1\% compared with $78.3 \pm 5.5 \%$, respectively. ${ }^{53}$ Another CNN performed better than other ML methods in predicting final infarct volume by incorporating MR imaging DWI, MRP, and FLAIR data, with an AUC of $0.88 \pm 0.12 .^{54}$ This CNN could predict tissue fate based on whether intravenous tissue plasminogen activator was administered, showing significantly different final infarct volumes $(P=.048){ }^{54}$ A CNN based on MRP source images was able to predict final infarct volume with an AUC of $0.871 \pm 0.024 .{ }^{55} \mathrm{~A}$ multicenter study showed that an attentiongated U-Net DL algorithm with DWI and MRP as inputs could predict final infarct volume regardless of reperfusion status, with a median AUC of 0.92 (IQR, 0.87-0.96) and significant overlap with the ground truth of a FLAIR sequence obtained 3-7 days after baseline presentation (Dice score, 0.53 ; IQR, 0.31-0.68). ${ }^{56}$

The e-ASPECTS software was able to predict poor clinical outcomes after thrombectomy (Spearman correlation $=-0.15$; $P=.027)$ and was an independent predictor of poor outcome in a multivariate analysis (OR, 0.79; 95\% CI, 0.63-0.99) while also demonstrating high consensus with 3 expert ASPECTS readers (ICC $=0.72,0.74$, and 0.76). ${ }^{57}$ Traditional ML techniques combining clinical data and core-penumbra mismatch ratio derived from MR imaging and MRP to determine postthrombolysis clinical outcomes performed with an AUC of 0.863 (95\% CI, 0.7740.951) for short-term (day 7) outcomes and 0.778 (95\% CI,
0.668-0.888) for long-term (day 90) outcomes. ${ }^{58}$ Decision treebased algorithms including extreme gradient boosting and gradient boosting machine were able to predict 90-day modified Rankin scale (mRS) $>2$ using imaging and clinical data with AUC of 0.746 (extreme gradient boosting) and 0.748 (gradient boosting machine), and performance improved when incorporating NIHSS at 24 hours and recanalization outcomes. ${ }^{59}$ ML techniques, including regularized logistic regression, linear SVM, and $\mathrm{RF}$, outperformed existing pretreatment scoring methods in predicting good clinical outcomes (mRS $\leq 2$ at 90 days) of patients with LVO who will undergo thrombectomy, with AUC 0.85-0.86 for ML models compared with $0.71-0.77$ for pretreatment scores. ${ }^{60}$ A combination CNN and ANN approach incorporating clinical and NCCT data predicted functional thrombolysis outcomes with accuracy 0.71 for 24-hour NIHSS improvement of $\geq 4$ and accuracy 0.74 for 90 -day mRS of $0-1 .^{61}$ Finally, traditional ML techniques and neural networks were used to predict hemorrhagic transformation of acute ischemic stroke before treatment from MRP source images and DWI, with the highest AUC of $0.837 \pm 2.6 \%$ using a kernel spectral regression ML technique. ${ }^{62}$ One limitation of this study was the variable recanalization of the participants, which may have confounded results.

\section{AI EVALUATION OF HEMORRHAGE}

This section focuses primarily on DL methods that have been used for intracranial hemorrhage (ICH) detection and classification, quantification, and prognostication (Online Table 5).

\section{Detection and Classification}

A study using two 2D convolutional neural networks, GoogLeNet and AlexNet, to detect basal ganglia hemorrhages on NCCT found that GoogLeNet with augmented data in a pretrained network was the most accurate $(A U C=1.0$; sensitivity and specificity $=100 \%)$ compared with the highest performing augmented, untrained AlexNet (AUC = 0.95; sensitivity $=100 \%$; and specificity $=80 \%) .{ }^{63}$ False positive results from basal ganglia calcification were seen in some of the methods, and sensitivity of detection of small basal ganglia hemorrhages remains to be investigated.

One of the largest cohorts for detection and classification of ICH examined more than 30,0000 NCCTs from different hospitals in India using DL algorithms. ${ }^{64}$ The algorithm performed well on 2 different validation datasets, Qure25k and CQ500, achieving AUCs of 0.92 (95\% CI, 0.91-0.93) and 0.94 (CI, 0.920.97), respectively, for detecting ICH. The algorithm was also able to classify subtypes of hemorrhage (parenchymal, intraventricular, subdural, extradural/epidural, and subarachnoid) with AUCs ranging from 0.90 to 0.96 for the Qure25K dataset and 0.93 to 0.97 for the CQ500 dataset. An additional feature of the algorithm was its ability to recognize associated pertinent CT findings, such as calvarial fracture, midline shift, and mass effect.

Another study using a fully 3D CNN with a large patient cohort was able to detect ICH and reprioritize studies as "stat" (defined as a positive ICH study) versus "routine." ${ }^{\prime 65}$ The AUC 
was 0.846 (95\% CI, 0.837-0.856), specificity was 0.80 (0.790$0.809)$, and sensitivity was $0.73(0.713-0.748)$. The algorithm was integrated into the radiologist's workflow, and time to detection was reduced from 512 to 19 minutes.

An explainable pretrained 2D convolutional neural networks system performed at a similar level to expert neuroradiologists on a relatively small cohort of cases when detecting acute ICH and classifying the $5 \mathrm{ICH}$ subtypes on NCCT. ${ }^{66}$ The algorithm incorporated techniques such as attention maps and prediction based modules to help mitigate the "black box" of the DL system. The system displayed a robust performance when detecting ICH on a retrospective dataset of 200 cases (AUC $=0.99$; sensitivity $=$ $98 \%$; and specificity $=95 \%$ ) and prospective dataset of 196 cases $($ AUC $=0.96 ;$ sensitivity $=92 \%$; and specificity $=95 \%)$. Furthermore, the overall localization accuracy of the attention maps was $78.1 \%$ compared with bleeding points annotated by expert neuroradiologists.

\section{Quantification}

A custom DL-trained hybrid 3D-2D CNN was able to detect and quantify ICHs on NCCT in a retrospective training cohort and a prospective testing cohort from the emergency department. ${ }^{67}$ Accuracy, AUC, sensitivity, specificity, positive predictive value, and negative predictive value for ICH detection for the training cohort were $0.975,0.983,0.971,0.975,0.793$, and 0.997 , respectively, and for the prospective cohort were 0.970, 0.981, 0.951, $0.973,0.829$, and 0.993 . For ICH quantification, Dice scores were $0.931,0.863$, and 0.772 , and Pearson correlation coefficients were $0.999,0.987$, and 0.953 for intraparenchymal hemorrhage, epidural or subdural hemorrhage, and $\mathrm{SAH}$, respectively, compared with semiautomated segmentation by a radiologist. This study used real-life prospective testing of the algorithm and quantified hemorrhage volume during segmentation. The study also addresses the black box critique with the use of a custom mask ROI-based CNN architecture.

A patch-based fully DL CNN simultaneously classified and quantified hemorrhages at a level equal to or above that of expert radiologists $(\mathrm{AUC}=0.991 \pm 0.006) .{ }^{68}$ The algorithm was able to identify some small hemorrhages that were missed by radiologists and performed well on a relatively small dataset. The strongly supervised approach took into account the heterogeneous morphology of hemorrhages and showed perfect sensitivity (1.00) while maintaining high specificity (0.87).

\section{Prognostication}

Identifying patients at risk for ICH expansion is important for prognostication. One study showed good performance when applying a SVM that incorporated various clinical and imaging variables to predict hematoma expansion on NCCT (AUC = 0.89 ; mean sensitivity $=81.3 \%$; and mean specificity $=84.8 \%) .{ }^{69}$ Rapid and accurate identification of ICH by AI methods could aid with triaging of positive studies.

\section{CONCLUSIONS}

Prompt detection and treatment of acute cerebrovascular disease is critical to reduce morbidity and mortality. The current application of AI in this field has allowed for vast opportunities to improve treatment selection and clinical outcomes by aiding in all parts of the diagnostic and treatment pathway, including detection, triage, and outcome prediction. Future studies validating AI techniques are needed to allow for more widespread use in various practice environments.

\section{ACKNOWLEDGMENTS}

The authors thank Aidoc, Avicenna, Brainomix, RapidAI, and Viz.ai for providing information regarding commercially available products and sample images of their applications for publication.

Disclosures: Daniel Chow—RELATED: Grant: Avicenna.ai*; UNRELATED: Consultancy: Canon Medical; Expert Testimony: Cullins \& Grandy; Grants/Grants Pending: Canon Medical, Novocure; Stock/Stock Options: Avicenna.ai. Christopher FilippiUNRELATED: Consultancy: Guerbet, Syntactx, Comments: Advisor on Al (Guerbet) and interpret brain MR for clinical trials (Syntactx); Grants/Grants Pending: FASNR grant and National MS Society grant; Stock/Stock Options: Minority stakeholder in start-up Avicenna. Wengui Yu-UNRELATED: Employment: University of California Irvine. Peter Chang-UNRELATED: Consultancy: Canon Medical, Comments: Consulting, travel expenses, honorarium for invited keynote speaker (RSNA); Stock/ Stock Options: Avicenna.ai, Comments: Co-founder, board member. *Money paid to institution.

\section{REFERENCES}

1. Ovbiagele B, Nguyen-Huynh MN. Stroke epidemiology: advancing our understanding of disease mechanism and therapy. Neurotherapeutics 2011;8:319-29 CrossRef Medline

2. Donkor ES. Stroke in the 21(st) century: a snapshot of the burden, epidemiology, and quality of life. Stroke Res Treat 2018;2018: 3238165 CrossRef Medline

3. Boehme AK, Esenwa C, Elkind MSV. Stroke risk factors, genetics, and prevention. Circ Res 2017;120:472-95 CrossRef Medline

4. Lee EJ, Kim YH, Kim N, et al. Deep into the brain: artificial intelligence in stroke imaging. J Stroke 2017;19:277-85 CrossRef Medline

5. Goodfellow I, Bengio Y, Courville A. Deep Learning. The MIT Press 2016

6. Saeys Y, Inza I, Larrañaga P. A review of feature selection techniques in bioinformatics. Bioinformatics 2007;23:2507-17 CrossRef Medline

7. Dreiseitl S, Ohno-Machado L. Logistic regression and artificial neural network classification models: a methodology review. J Biomed Inform 2002;35:352-59 CrossRef Medline

8. Wang S, Summers RM. Machine learning and radiology. Med Image Anal 2012;16:933-51 CrossRef Medline

9. Jordan MI, Mitchell TM. Machine learning: trends, perspectives, and prospects. Science 2015;349:255-60 CrossRef Medline

10. He K, Zhang X, Ren S, et al. Deep residual learning for image recognition. arXiv:1512:03385;10 Dec 2015;1-12

11. Krizhevsky A, Sutskever I, Hinton G. Imagenet classification with deep convolutional neural networks. Proc Advances in Neural Information Processing Systems 2012;1090-98

12. Krizhevsky A, Sutskever I, Hinton GE. Imagenet classification with deep convolutional neural networks. Neural information processing systems. Stateline 2012;1097-1105

13. Setio AA, Ciompi F, Litjens G, et al. Pulmonary nodule detection in CT images: false positive reduction using multi-view convolutional networks. IEEE Trans Med Imaging 2016;35:1160-69 CrossRef Medline

14. Roth HR, Lu L, Liu J, et al. Improving computer-aided detection using convolutional neural networks and random view aggregation. IEEE Trans Med Imaging 2016;35:1170-81 CrossRef Medline

15. Qi D, Hao C, Lequan Y, et al. Automatic detection of cerebral microbleeds from MR images via 3D convolutional neural networks. IEEE Trans Med Imaging 2016;35:1182-95 CrossRef Medline 
16. Zou KH, Warfield SK, Bharatha A, et al. Statistical validation of image segmentation quality based on a spatial overlap index1: scientific reports. Acad Radiol 2004;11:178-89 CrossRef

17. Yamashita R, Nishio M, Do RKG, et al. Convolutional neural networks: an overview and application in radiology. Insights Imaging 2018;9:611-29 CrossRef Medline

18. Murray NM, Unberath M, Hager GD, et al. Artificial intelligence to diagnose ischemic stroke and identify large vessel occlusions: a systematic review. J Neurointerv Surg 2020;12:156-64 CrossRef

19. Liew S-L, Anglin JM, Banks NW, et al. A large, open source dataset of stroke anatomical brain images and manual lesion segmentations. Sci Data 2018;5:180011 CrossRef Medline

20. Chilamkurthy S, Ghosh R, Tanamala S, et al. Development and validation of deep learning algorithms for detection of critical findings in head CT scans. arXiv :1803:05854;30 March 2018; 1-18

21. Flanders AE, Prevedello LM, Shih G, et al. Construction of a machine learning dataset through collaboration: the RSNA 2019 brain CT hemorrhage challenge. Radiol Art Int 2020;2:e190211 CrossRef

22. Winzeck S, Hakim A, McKinley R, et al. ISLES 2016 and 2017benchmarking ischemic stroke lesion outcome prediction based on multispectral MRI. Front Neurol 2018;9:679 CrossRef Medline

23. Clèrigues A, Valverde $S$, Bernal J, et al. Acute ischemic stroke lesion core segmentation in CT perfusion images using fully convolutional neural networks. Comput Biol Med 2019;115:103487 CrossRef Medline

24. American Society of Functional Neuroradiology. ASFNR Ai challenge. https://aichallenge.asfnr.org. Accessed July 5, 2020

25. Nogueira RG, Jadhav AP, Haussen DC, et al. Thrombectomy 6 to 24 hours after stroke with a mismatch between deficit and infarct. $N$ Engl J Med 2018;378:11-21 CrossRef Medline

26. Saver JL, Goyal M, Bonafe A, et al. Stent-retriever thrombectomy after intravenous t-PA vs. t-PA alone in stroke. N Engl J Med 2015; 372:2285-95 CrossRef Medline

27. Albers GW, Marks MP, Kemp S, et al. Thrombectomy for stroke at 6 to 16 hours with selection by perfusion imaging. $N$ Engl J Med 2018;378:708-18 CrossRef Medline

28. Campbell BCV, Mitchell PJ, Kleinig TJ, et al. Endovascular therapy for ischemic stroke with perfusion-imaging selection. $N$ Engl J Med 2015;372:1009-18 CrossRef Medline

29. Wismüller A, Stockmaster L. A prospective randomized clinical trial for measuring radiology study reporting time on artificial Intelligence-based detection of intracranial hemorrhage in emergent care head CT. arXiv :2002:12515; 28 Feb 2020; 1-7

30. Tang FH, Ng DK, Chow DH. An image feature approach for computer-aided detection of ischemic stroke. Comput Biol Med 2011; 41:529-36 CrossRef Medline

31. Abedi V, Goyal N, Tsivgoulis G, et al. Novel screening tool for stroke using artificial neural network. Stroke 2017;48:1678-81 CrossRef Medline

32. Chen L, Bentley P, Rueckert D. Fully automatic acute ischemic lesion segmentation in DWI using convolutional neural networks. NeuroImage Clin 2017;15:633-43 CrossRef Medline

33. Guerrero R, Qin C, Oktay O, et al. White matter hyperintensity and stroke lesion segmentation and differentiation using convolutional neural networks. NeuroImage Clin 2018;17:918-34 CrossRef Medline

34. Oman O, Makela T, Salli E, et al. 3D convolutional neural networks applied to CT angiography in the detection of acute ischemic stroke. Eur Radiol Exp 2019;3:8 CrossRef Medline

35. Kasasbeh AS, Christensen S, Parsons MW, et al. Artificial neural network computer tomography perfusion prediction of ischemic core. Stroke 2019;50:1578-81 CrossRef Medline

36. Qiu W, Kuang H, Teleg E, et al. Machine learning for detecting early infarction in acute stroke with non-contrast-enhanced CT. Radiology 2020;294:638-44 CrossRef Medline
37. Takahashi N, Lee Y, Tsai D-Y, et al. An automated detection method for the MCA dot sign of acute stroke in unenhanced CT. Radiol Phys Technol 2014;7:79-88 CrossRef Medline

38. Chen Z, Zhang R, Xu F, et al. Novel prehospital prediction model of large vessel occlusion using artificial neural network. Front Aging Neurosci 2018;10:181 CrossRef Medline

39. Chatterjee A, Somayaji NR, Kabakis IM. Abstract WMP16: artificial intelligence detection of cerebrovascular large vessel occlusionnine month, 650 patient evaluation of the diagnostic accuracy and performance of the Viz.ai LVO algorithm. Stroke 2019;50: AWMP16 CrossRef

40. Barreira C, Bouslama M, Lim J, et al. E-108 Aladin study: automated large artery occlusion detection in stroke imaging study-a multicenter analysis. J Neurointerv Surg 2018;10:A101-02 CrossRef

41. Goyal M, Demchuk AM, Menon BK, et al. Randomized assessment of rapid endovascular treatment of ischemic stroke. $N$ Engl J Med 2015;372:1019-30 CrossRef Medline

42. Jovin TG, Chamorro A, Cobo E, et al. Thrombectomy within 8 hours after symptom onset in ischemic stroke. $N$ Engl J Med 2015;372:2296-2306 CrossRef Medline

43. Nagel S, Sinha D, Day D, et al. e-ASPECTS software is non-inferior to neuroradiologists in applying the ASPECT score to computed tomography scans of acute ischemic stroke patients. Int J Stroke 2017;12:615-22 CrossRef Medline

44. Guberina N, Dietrich U, Radbruch A, et al. Detection of early infarction signs with machine learning-based diagnosis by means of the Alberta Stroke Program Early CT score (ASPECTS) in the clinical routine. Neuroradiology 2018;60:889-901 CrossRef Medline

45. Kuang H, Najm M, Chakraborty D, et al. Automated ASPECTS on noncontrast CT scans in patients with acute ischemic stroke using machine learning. AJNR Am J Neuroradiol 2019;40:33-38 CrossRef Medline

46. Maegerlein C, Fischer J, Monch S, et al. Automated calculation of the Alberta Stroke Program Early CT score: feasibility and reliability. Radiology 2019;291:141-48 CrossRef Medline

47. Albers GW, Wald MJ, Mlynash M, et al. Automated calculation of Alberta Stroke Program Early CT score: validation in patients with large hemispheric infarct. Stroke 2019;50:3277-79 CrossRef Medline

48. Grunwald IQ, Kulikovski J, Reith W, et al. Collateral automation for triage in stroke: evaluating automated scoring of collaterals in acute stroke on computed tomography scans. Cerebrovasc Dis 2019;47:217-22 CrossRef Medline

49. Wang K, Shou Q, Ma SJ, et al. Deep learning detection of penumbral tissue on arterial spin labeling in stroke. Stroke 2020;51:489-97 CrossRef Medline

50. Ho KC, Speier W, Zhang H, et al. A machine learning approach for classifying ischemic stroke onset time from imaging. IEEE Trans Med Imaging 2019;38:1666-76 CrossRef Medline

51. Lee H, Lee E-J, Ham S, et al. Machine learning approach to identify stroke within 4.5 hours. Stroke 2020;51:860-66 CrossRef Medline

52. Wu O, Koroshetz WJ, Østergaard L, et al. Predicting tissue outcome in acute human cerebral ischemia using combined diffusion- and perfusion-weighted MR imaging. Stroke 2001;32:933-42 CrossRef Medline

53. Stier N, Vincent N, Liebeskind D, et al. Deep learning of tissue fate features in acute ischemic stroke. Proceedings (IEEE Int Conf Bioinformatics Biomed) 2015;2015:1316-21 CrossRef Medline

54. Nielsen A, Hansen MB, Tietze A, et al. Prediction of tissue outcome and assessment of treatment effect in acute ischemic stroke using deep learning. Stroke 2018;49:1394-1401 CrossRef Medline

55. Ho KC, Scalzo F, Sarma K, et al. Predicting ischemic stroke tissue fate using a deep convolutional neural network on source magnetic resonance perfusion images. J Med Imag 2019;6:1 CrossRef Medline

56. Yu Y, Xie Y, Thamm T, et al. Use of deep learning to predict final ischemic stroke lesions from initial magnetic resonance imaging. JAMA Netw Open 2020;3:e200772 CrossRef Medline 
57. Pfaff J, Herweh C, Schieber S, et al. e-ASPECTS correlates with and is predictive of outcome after mechanical thrombectomy. AJNR Am J Neuroradiol 2017;38:1594-99 CrossRef Medline

58. Tang T-Y, Jiao Y, Cui Y, et al. Development and validation of a penumbra-based predictive model for thrombolysis outcome in acute ischemic stroke patients. EBioMedicine 2018;35:251-59 CrossRef Medline

59. Xie Y, Jiang B, Gong E, et al. Use of gradient boosting machine learning to predict patient outcome in acute ischemic stroke on the basis of imaging, demographic, and clinical information. AJR Am J Roentgenol 2019;212:44-51 CrossRef Medline

60. Nishi H, Oishi N, Ishii A, et al. Predicting clinical outcomes of large vessel occlusion before mechanical thrombectomy using machine learning. Stroke 2019;50:2379-88 CrossRef Medline

61. Bacchi S, Zerner T, Oakden-Rayner L, et al. Deep learning in the prediction of ischaemic stroke thrombolysis functional outcomes: a pilot study. Acad Radiol 2020;27:e19-23 CrossRef Medline

62. Yu Y, Guo D, Lou M, et al. Prediction of hemorrhagic transformation severity in acute stroke from source perfusion MRI. IEEE Trans Biomed Eng 2018;65:2058-65 CrossRef Medline

63. Desai V, Flanders A, Lakhani P. Application of deep learning in neuroradiology: automated detection of basal ganglia hemorrhage using 2D-convolutional neural networks. arXiv :1710:03823;10 Oct 2017;1-7
64. Chilamkurthy S, Ghosh R, Tanamala S, et al. Deep learning algorithms for detection of critical findings in head CT scans: a retrospective study. Lancet 2018;392:2388-96 CrossRef Medline

65. Arbabshirani MR, Fornwalt BK, Mongelluzzo GJ, et al. Advanced machine learning in action: identification of intracranial hemorrhage on computed tomography scans of the head with clinical workflow integration. NPJ Digital Med 2018;1:9 CrossRef Medline

66. Lee H, Yune S, Mansouri M, et al. An explainable deep-learning algorithm for the detection of acute intracranial haemorrhage from small datasets. Nat Biomed Eng 2019;3:173-82 CrossRef Medline

67. Chang PD, Kuoy E, Grinband J, et al. Hybrid 3D/2D convolutional neural network for hemorrhage evaluation on head CT. AJNR Am J Neuroradiol 2018;39:1609-16 CrossRef Medline

68. Kuo W, Häne C, Mukherjee P, et al. Expert-level detection of acute intracranial hemorrhage on head computed tomography using deep learning. Proc Natl Acad Sci USA 2019;116:22737-45 CrossRef Medline

69. Liu J, Xu H, Chen Q, et al. Prediction of hematoma expansion in spontaneous intracerebral hemorrhage using support vector machine. EBioMedicine 2019;43:454-59 CrossRef Medline 